\title{
Reply on the comments about Piccione F, Maccarone MC, Cortese AM, Rocca G, Sansubrino U, Piran G, Masiero S. Rehabilitative management of pelvic fractures: a literature-based update. Eur J Transl Myol. 2021 Sep 17;31(3):9933. doi: 10.4081/ejtm.2021.9933
}

\author{
Maria Chiara Maccarone (1), Daniele Coraci (2), Umberto Sansubrino (2), Francesco \\ Piccione (2), Stefano Masiero (2), Raffaele Zanella (1).
}

(1) Physical Medicine and Rehabilitation School, University of Padova, Padua, Italy; Department of Neuroscience, Rehabilitation Unit, University of Padova, Padova, Italy.

This article is distributed under the terms of the Creative Commons Attribution Noncommercial License (CC BY-NC 4.0) which permits any noncommercial use, distribution, and reproduction in any medium, provided the original author(s) and source are credited.

\begin{abstract}
We thank the authors of the Letter of Zehua Li, et al. (2021) ${ }^{1}$ and the Editor for the opportunity to discuss our work more thoroughly. The comments on the article written by Piccione et al. $(2021)^{2}$ are certainly interesting and highlight limitations of the previously published article. However, we need to make some clarifications.
\end{abstract}

Key Words: Pelvic; acetabular; fractures; rehabilitation.

Eur J Transl Myol 31 (4): 103072021 doi: 10.4081/ejtm.2021.10307

We thank the authors of the above Letter of Zehua Li, et al. (2021) ${ }^{1}$ and the Editor for the opportunity to discuss our work more thoroughly. The above comments on the article written by Piccione et al. (2021) ${ }^{2}$ are certainly interesting and highlight limitations of the previously published article.

However, we need to make some clarifications. A narrative review, using MEDLINE, EMBASE, and the Cochrane Database of Systematic Reviews, was conducted selecting papers with abstracts written in English and published between January 1980 to May 2020. First of all, the choice of not conducting a review of the grey literature lies in the fact that often this type of studies and publications do not report peer-reviewed evidence.

Since we had to give an update of the rehabilitation indications for patients with pelvic fractures (PFs), we considered it was appropriate to investigate more relevant papers from a scientific point of view, accessible through the main research databases. We selected studies with abstracts available that could be easily consulted by readers interested in deepening the topic.

Since the PFs impact is very broad but the rehabilitation aspects are poorly addressed, we chose to consider articles not only purely related to rehabilitative interventions. So, we selected manuscripts addressing the outcomes of PFs in multiple medical fields.
We therefore consulted databases without restriction of medical domain so as to include as many articles as possible. Secondly, since the review of Piccione et al. $(2021)^{1}$ is not a systematic review but rather an update manuscript or a narrative review, we think that the grading criteria should not follow a predefined coding. Without claiming to conduct a systematic review, we have given an indication of the relevance of the selected articles based on the methods used to conduct the studies (high quality: randomized controlled trials, metaanalysis of randomized controlled trials; moderate quality: not randomized trials, prospective cohort studies; low quality: retrospective observational, retrospective cohort, and case-control studies; very low quality: case series, case reports, reviews, other). According to these simple criteria, only the studies on associated traumatic injuries and PFs sequelae, as well as on the treatment of complex PFs in the post-traumatic period were judged to be of good quality (one manuscript per type). There were also studies of moderate quality on the same issues. In the other areas, research of moderate quality was found, particularly in the area of male sexual dysfunction. However, the majority of the references for the other queries were poor or of extremely low quality research. Despite its importance related to the population impact, it is clear that the topic requires additional investigation through high-quality clinical trials. 


\section{Rehabilitation of pelvic fractures needs to be further optimized}

Eur J Transl Myol 31 (4): 10307, 2021 doi: 10.4081/ejtm.2021.10307

Therefore, we agree that there is a need for clarity in the area of pelvic fracture rehabilitation, which still has many unclear points. However, we have tried to make an update of the literature published in the last forty years, so as to summarize the knowledge acquired so far.

\section{List of acronyms}

PFs - Pelvic fractures

\section{Authors contributions}

All authors were involved in drafting the manuscript and in its critical revision. All authors read and approved the final manuscript.

\section{Acknowledgments}

None.

\section{Funding}

None.

\section{Conflict of Interest}

The authors declare no competing interests.

\section{Ethical Publication Statement}

We confirm that we have read the Journal's position on issues involved in ethical publication and affirm that this report is consistent with those guidelines.

\section{Corresponding Author}

Maria Chiara Maccarone, Physical Medicine and Rehabilitation School, University of Padova, Via Giustiniani 3, 35128 Padova, Italy

ORCID iD: 0000-0001-6509-5707

E-mail: mariachiara.maccarone@studenti.unipd.it

E-mails and ORCID iD of co-authors

Daniele Coraci: daniele.coraci@unipd.it

ORCID iD: 0000-0002-7019-9006

Umberto Sansubrino: umberto.sansubrino@aopd.veneto.it

ORCID iD: 0000-0003-1453-1534

Francesco Piccione: francesco.piccione@aopd.veneto.it ORCID iD: 0000-0002-0173-903X

Stefano Masiero: stef.masiero@unipd.it

ORCID iD: 0000-0002-0361-4898

Raffaele Zanella: raffaele.zanella@studenti.unipd.it

ORCID iD: 0000-0003-3152-9348

\section{References}

1. Piccione F, Maccarone MC, Cortese AM, Rocca G, Sansubrino U, Piran G, Masiero S. Rehabilitative management of pelvic fractures: a literature-based update. Eur J Transl Myol. 2021 Sep 17;31(3):9933. doi: 10.4081/ejtm.2021.9933.

2. Li Z, Maimaitiming D, Sun L, Wang H, Xiong W Rehabilitative management of pelvic fractures needs to be further optimized Eur J Transl Myol. 202131(3):9933. doi: 10.4081/ejtm.2021.9933.

Submission: October 30, 2021 Accepted for publication: October 30, 2021 